\title{
Magnetic blue shift of Mott gaps enhanced by double exchange
}

\author{
Mohsen Hafez-Torbati $\odot,{ }^{1, *}$ Davide Bossini $\odot,{ }^{2, \dagger}$ Frithjof B. Anders $\odot,{ }^{3, \star}$ and Götz S. Uhrig $\odot^{1, \S}$ \\ ${ }^{1}$ Lehrstuhl für Theoretische Physik I, Technische Universität Dortmund, Otto-Hahn-Straße 4, 44221 Dortmund, Germany \\ ${ }^{2}$ Department of Physics and Center for Applied Photonics, University of Konstanz, D-78457 Konstanz, Germany \\ ${ }^{3}$ Lehrstuhl für Theoretische Physik II, Technische Universität Dortmund, Otto-Hahn-Straße 4, 44221 Dortmund, Germany
}

(Received 7 December 2020; revised 17 December 2021; accepted 20 December 2021; published 30 December 2021)

\begin{abstract}
A substantial energy gap of charge excitations induced by strong correlations is the characteristic feature of Mott insulators. We study how the Mott gap is affected by long-range antiferromagnetic order. Our key finding is that the Mott gap is increased by the magnetic ordering: A magnetic blue shift (MBS) occurs. Thus the effect is proportional to the exchange coupling in the leading order in the Hubbard model. In systems with additional localized spins the double-exchange mechanism induces an additional contribution to the MBS which is proportional to the hopping in the leading order. The coupling between spin and charge degrees of freedom bears the potential to enable spin-to-charge conversion in Mott systems on extreme time scales determined by hopping and exchange only, since a spin-orbit-mediated transfer of angular momentum is not involved in the process. In view of spintronic and magnonic applications, it is highly promising to observe that several entire classes of compounds show exchange and double-exchange effects. Exemplarily, we show that the magnetic contribution to the band-gap blue shift observed in the optical conductivity of $\alpha$-MnTe is correctly interpreted as the MBS of a Mott gap.
\end{abstract}

DOI: 10.1103/PhysRevResearch.3.043232

\section{INTRODUCTION}

The discovery of insulating behavior in transition metal oxides [1] and its explanation in terms of strong electronelectron interaction [2] were the origin of the very active research field of strongly correlated systems [3-6]. The low-energy physics of Mott insulators is governed by spin excitations [7]. A common and successful strategy to treat them consists in disentangling spin and charge degrees of freedom [8-11]. However, following this approach, it is difficult to track the coupling between charge and spin dynamics, which is expected to play a pivotal role in the recent massive surge of interest in antiferromagnetic (AFM) spintronics [12-17]. The grand goal of this impressive research effort consists in establishing the ability to convert spin signals into charge responses on the shortest possible time scale and minimizing as much as possible the energy dissipations.

So far, the typical route to spintronics relies on spin-orbitbased transport effects [12] requiring a heavy metal layer on top of the antiferromagnet to read out the electric system. The vision of a spintronic information technology based solely on

\footnotetext{
*mohsen.hafez@tu-dortmund.de

†davide.bossini@uni-konstanz.de

†rithjof.anders@tu-dortmund.de

§goetz.uhrig@tu-dortmund.de
}

Published by the American Physical Society under the terms of the Creative Commons Attribution 4.0 International license. Further distribution of this work must maintain attribution to the author(s) and the published article's title, journal citation, and DOI. antiferromagnetism thus completely relies on the strength of the spin-orbit coupling, which defines both the spin-charge conversion efficiency and the operational frequency of a device. Generically, exchange couplings are larger than spinorbit couplings by at least one order of magnitude. This makes it desirable to use effects of purely exchange origin implying shorter characteristic time scales and hence higher operational frequencies.

Evidence has been reported that the charge gap in a Mott insulator, the Mott gap, depends on the magnetic ordering [18-20]. In particular, a magnetic shift of the band gap proportional to the square of the sublattice magnetization could enable a coherent modulation of the band-gap energy itself. Coherent dynamics of the order parameter in AFM insulators has been photoinduced and manipulated [21-23] where frequencies of $22 \mathrm{THz}$ [24-26] were found. This framework would enable a coherent manipulation of the transport properties at the unprecedented $20 \mathrm{THz}$ working frequency.

Local electronic interactions are essential for the formation of magnetic moments. Thus there are two possible dichotomous scenarios for the influence of the magnetic ordering on the charge gap: (i) The charge gap is a band gap of $s$ and $p$ electrons which are different from the electrons forming the magnetic degrees of freedom. Then, the influence of the localized spins is only indirect via superexchange with the itinerant electrons. (ii) The charge gap is a Mott gap, so that the electrons forming the localized spins are also the ones forming the charge gap. A charge-transfer insulator also belongs to scenario (ii) because one of the bands relevant for the optical gap is a strongly correlated one.

So far, the observed magnetic shifts have been discussed in terms of scenario (i) [27-29]. The obtained results and even 
the overall sign of the effect, red or blue shift, depend on many details of the system. Only very recently has the observed magnetic shift of the band gap in hexagonal MnTe $(\alpha-\mathrm{MnTe})$ $[30,31]$ been linked to strong local interactions in a local static mean-field model [30]. In this paper, we investigate the temperature dependence of the Mott gap across the transition from a paramagnetic to an AFM insulator. The transition to the ordered phase is accompanied by a noticeable increase in the Mott gap, i.e., a magnetic blue shift (MBS) of the Mott gap occurs.

We address the fundamental nature of this MBS. By studying models with increasing complexity within the dynamical mean-field theory (DMFT) [3] we are able to pinpoint the subtle differences and clarify the influence of the charge hopping and the magnetic exchange interaction. We apply our approach to a real material, $\alpha-\mathrm{MnTe}$, and demonstrate the very good agreement with the available experimental data.

First, we study the MBS in two fundamental models, the Hubbard model and the Hubbard-Kondo model, and unfold some generic features. In the Hubbard model, we show that in the leading order the MBS is proportional to the magnetic exchange appearing by mapping the half-filled Hubbard model to a Heisenberg model or to a $t-J$ model (at finite doping). This results from the Slater mechanism: As a static alternating field induced by the magnetic order opens an energy gap in a metal, such a field also increases the existing charge gap in a Mott insulator.

In the Hubbard-Kondo model an additional local spin $S$ is included at each lattice site to account for nonitinerant magnetic degrees of freedom. Due to Hund's rules the itinerant and the local spin are coupled locally by a ferromagnetic coupling $J_{\mathrm{H}}>0$. In this model, we find an additional contribution to the MBS which is proportional to the hopping matrix element. This hopping contribution is the dominant contribution in systems with a large Hubbard interaction, which implies a small magnetic exchange. We reveal that this contribution is induced by the double-exchange mechanism due to a reduced effective hopping upon transition from the paramagnetic to the AFM insulator.

Second, we verify our approach by analyzing an exemplary system promising for applications, hexagonal MnTe $(\alpha-$ $\mathrm{MnTe})$, which has been experimentally investigated [30,31]. We extend the $S=5 / 2$ spin model explaining the inelastic neutron scattering data $[32,33]$ to a Hubbard-Kondo model allowing for the coupling of the spin and charge degrees of freedom. We compute the MBS of the Mott gap of the half-filled $3 d$ shell of Mn ions. Using only generic parameters established for $\alpha$-MnTe in the literature and without any fine-tuning, we achieve an overall excellent description of the MBS measured in the optical conductivity. We unveil the origin of the MBS in $\alpha$-MnTe data and find a magnetic exchange contribution of $36 \%$ and a hopping contribution of $64 \%$. Our findings set the stage to study coupled spin and charge dynamics in strongly correlated systems, including the specific case of $\alpha$-MnTe.

This paper is organized as follows. After this Introduction, results for the Hubbard model are shown and interpreted. Subsequently, the results for the Hubbard-Kondo model are presented and discussed, in particular, the additional contribution to the MBS stemming from the double-exchange mechanism. Section IV deals with the particular case of $\alpha$-MnTe as a candidate for significant spin-charge coupling based on the MBS. In Sec. V the results are summarized, and a brief outlook is given.

\section{THE THREE-DIMENSIONAL HUBBARD MODEL}

The Hubbard model [34] at half filling comprises hopping between nearest-neighbor (NN) sites controlled by the parameter $t$ and an interaction $U$ between electrons at the same site with opposite spins

$$
H_{\mathrm{H}}=-t \sum_{\langle i, j\rangle} \sum_{\sigma=\uparrow, \downarrow}\left(c_{j, \sigma}^{\dagger} c_{i, \sigma}+\text { H.c. }\right)+U \sum_{i} n_{i, \downarrow} n_{i, \uparrow} .
$$

This well-studied model shows a particle-hole symmetry at half filling with respect to the energy $\mu=U / 2$ defining the chemical potential $\mu$ used throughout this paper. The phase diagram at finite temperatures on the cubic lattice is well known at half filling [35-39]. At $T=0$, the ground state is a Néel antiferromagnet for any finite $U / t$ [40]. The Néel temperature $T_{\mathrm{N}}$ separating the paramagnetic and the AFM phases increases from 0 upon increasing $U$ [37], reaches a maximum, and decreases as $T_{\mathrm{N}} \propto t^{2} / U$ in the strong-coupling limit $U \gg t$ where the model can be mapped onto a spin-1/2 Heisenberg model. At high temperatures, the phase is a metal for small $U / t$ and a paramagnetic Mott insulator for large $U / t$ separated from the metallic phase by a crossover region. At large $U / t$, the Mott gap is proportional to $U$ as a charge excitation leads to the creation of a double occupancy which requires the energy $U$.

We define the bare charge gap $\Delta$ as the value of the charge gap in the absence of the hopping, $t=0$. Although in the Hubbard model the bare charge gap just equals the Hubbard interaction $U$, it remains the relevant quantity also in the Hubbard-Kondo model defining the magnetic exchange interaction $J=4 t^{2} / \Delta$. We use the bare charge gap $\Delta$ in cases where we aim to compare our results for the Hubbard model and the Hubbard-Kondo model.

We employ the DMFT [3] with exact diagonalization (ED) [41] as the impurity solver. This approach is well established for strong local interactions where subtle effects such as an emerging exponentially low energy scale associated with the formation of a narrow band at the chemical potential in the metallic phase cannot occur. For more details on the method we refer the reader to Appendix A. We compute the averaged local spectral function $A(\omega)=\left(A_{\sigma}^{A}(\omega)+A_{\sigma}^{B}(\omega)\right) / 2$ from the imaginary part of the local Green's function of sublattices $A$ and $B$. We point out that the spectral function $A(\omega)$ does not depend on spin even in the AFM phase because we average over both sublattices.

We plot the local spectral function $A(\omega)$ of the Hubbard model for different temperatures in the magnetic insulator (MI) phase in Fig. 1(a) and in the paramagnetic insulator (PI) phase in Fig. 1(b) for $U=20 t$. The spectral functions for the different parameters are shifted vertically for clarity. Note that the peak structure in $A(\omega)$ is caused by the discretized representation of the conduction band in the ED impurity solver with the number of bath sites $n_{b}=6$. Lowering the temperature $T$ in the PI hardly changes $A(\omega)$. However, in the MI, a shift of the electron and hole contributions to 


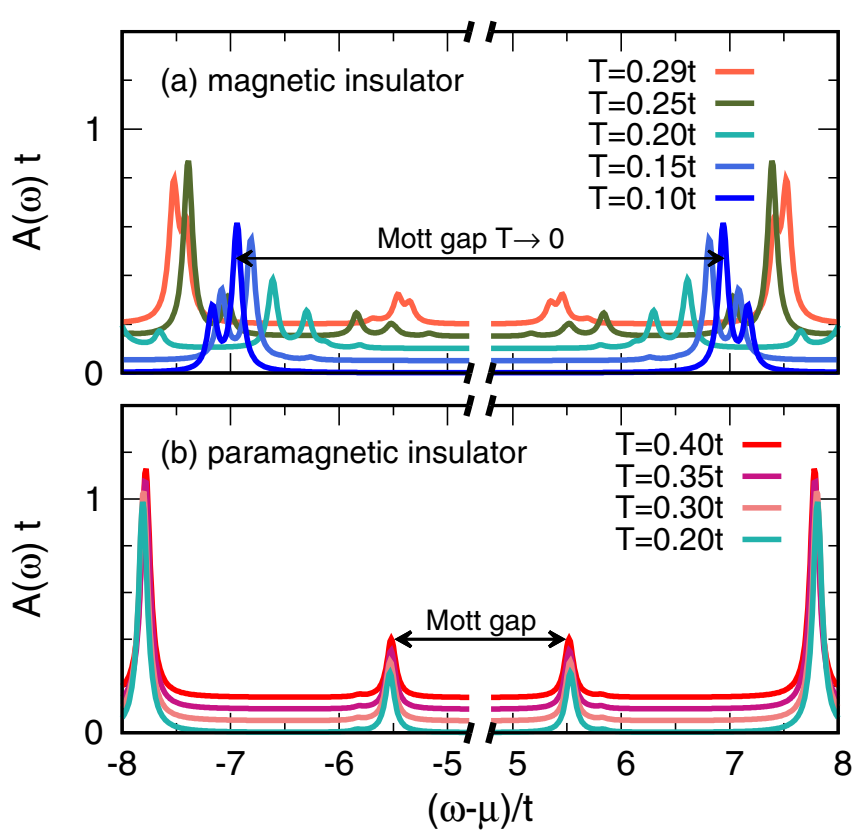

FIG. 1. Spectral function $A(\omega)$ vs $\omega$ in the range $[-8 t,+8 t]$ at various temperatures $T$ in the magnetic insulator (MI) (a) and in the paramagnetic insulator (PI) (b) for $U=20 t$ and $n_{b}=6$ bath sites in the impurity problem. The Néel temperature is given by $T_{\mathrm{N}} \approx 0.3 t$.

higher excitation energies is clearly observed. Below the Néel temperature $T_{\mathrm{N}} \approx 0.3 t$, the stable phase is the MI, but the metastable PI solution can be computed as well and was added to Fig. 1(b) for comparison.

The Mott gap is obtained from the energy difference between the two excitation energies of the spectrum that are closest to the chemical potential $\mu$; see the indicated arrows in Fig. 1. While this gap is apparently a constant in the PI phase as can be seen in Fig. 1(b), it shows a strong temperature dependency in the MI phase in Fig. 1(a). Upon reducing $T$, the electron and hole peaks at $\pm 5.5 t$ shift apart to $\pm 7 t$ due to the magnetic ordering. This leads to a MBS of the Mott gap $\Gamma_{\mathrm{MG}}(T)$ of about $3 t$ as $T \rightarrow 0$.

We depict the Mott gap as well as the local spin polarization $m$ in units of $\hbar$ vs the temperature $T$ for $U=15 t$ in Fig. 2(a) and for $U=20 t$ in Fig. 2(b). The results are displayed for two bath sizes, $n_{b}=6$ and $n_{b}=8$, to illustrate the accuracy of the approach. For $U=15 t$, the gap in the PI

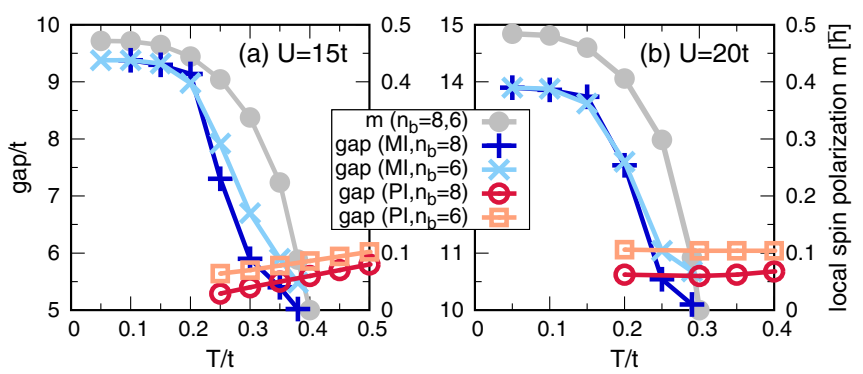

FIG. 2. The Mott gap in the magnetic insulator (MI) and in the paramagnetic insulator (PI) as a function of temperature for $U=15 t$ (a) and $U=20 t$ (b). The gray lines show the local spin polarization $m$ (right axes). The results for $n_{b}=6$ and $n_{b}=8$ are compared.

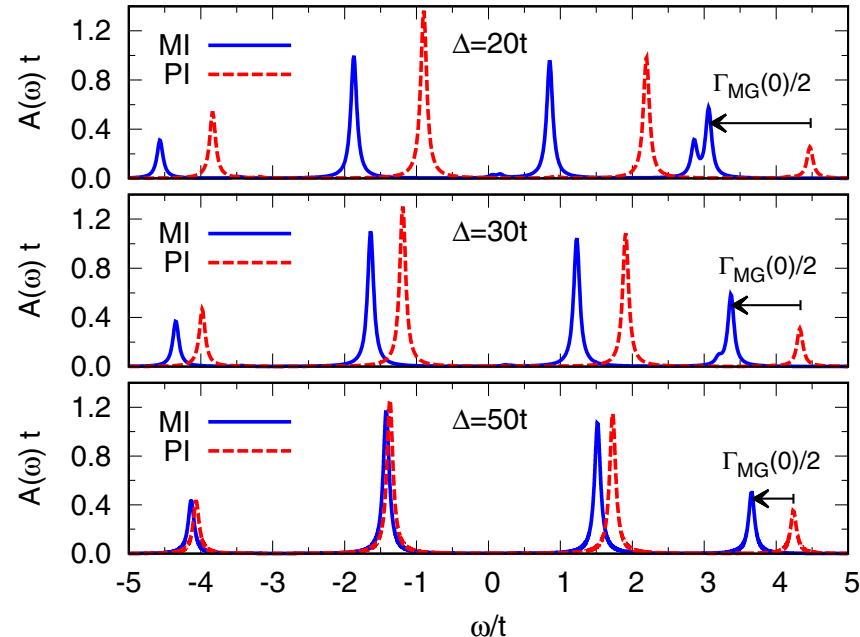

FIG. 3. The lower band of the spectral function in the Hubbard model for various values of the bare charge gap $\Delta=U$ in the MI close to $T=0$ and in the PI close to $T=T_{\mathrm{N}}$. The Fermi energy is located at $\omega=\mu=U / 2$. We have used the bare charge gap $\Delta$ as the label since we aim to compare the results with the results of the Hubbard-Kondo model. Clearly, the spectral functions in the PI and in the MI phase approach each other upon increasing $\Delta$. The results are obtained for $n_{b}=6$ bath sites in the impurity solver. The MBS of the Mott gap $\Gamma_{\mathrm{MG}}(0)$ is twice the indicated arrows.

decreases slowly upon lowering the temperature. For $U=20 t$ it remains almost constant. However, in both cases there is a rapid increase in the gap upon entering the MI phase which illustrates the MBS.

At the continuous transition from the PI to the MI the gaps have to be equal. This is not quite the case, most likely because of inaccuracies in extracting the gap from the ED data at finite bath sites. For $U=15 t$, the gap value is about $5.5 t$ close to the transition temperature and rises to about $9.4 t$ for $T \rightarrow 0$. Comparing Figs. 2(a) and 2(b), a decrease in the MBS upon increasing $U$ from $15 t$ to $20 t$ is observed. Such a decrease in the MBS in the Mott regime has also been observed in Ref. [20].

To analyze the MBS $\Gamma_{\mathrm{MG}}(T)$ near $T=0$ further, we depict the spectral function as a function of $\omega$ for various values of the bare charge gap $\Delta=U$ in the MI close to $T=0$ and in the PI close to $T=T_{\mathrm{N}}$ in Fig. 3. We have used the bare charge gap $\Delta$ as the label since we aim to compare the results with the results of the Hubbard-Kondo model in the next section. Only the lower Hubbard band is shown in Fig. 3 because the upper Hubbard band is its mirror image with respect to $\omega=\mu=U / 2$ due to electron-hole symmetry, which is perfectly realized in our numerical data. We indicated the shift of the excitation peak closest to the chemical potential by an arrow defining half of the MBS, $\Gamma_{\mathrm{MG}}(0) / 2$. The results are for $n_{b}=6$ bath sites in the impurity solver. Figure 3 reveals that the spectral functions in the MI and in the PI approach each other more and more upon increasing the bare charge gap $\Delta=U$. Consequently, the MBS $\Gamma_{\mathrm{MG}}(0)$ decreases as $t / \Delta \rightarrow 0$.

The MBS in the Hubbard model has already been observed in previous work $[18,19]$, but not systematically studied. 


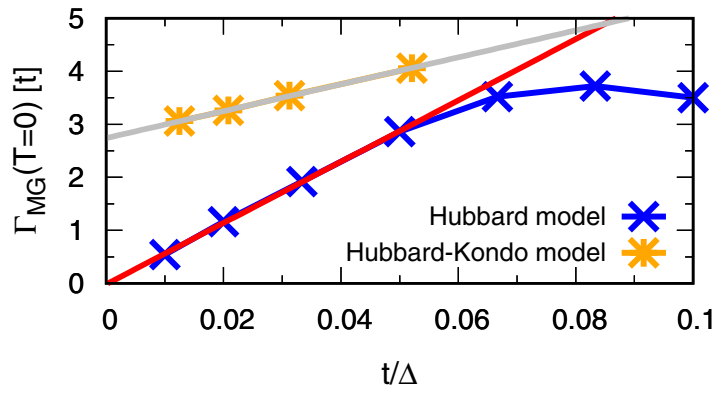

FIG. 4. The magnetic blue shift of the Mott gap $\Gamma_{\mathrm{MG}}(T)$ in units of the hopping $t$ at $T=0$ is plotted vs $t / \Delta$, where $\Delta$ is the bare charge gap. The results are obtained for $n_{b}=6$ bath sites in the impurity problem. In the Hubbard-Kondo model we set the local spin to $S=2$ and the Hund coupling to $J_{\mathrm{H}}=0.15 U$.

Recently, its monotonic decrease in the Mott regime for $U \rightarrow$ $\infty$ was noted in Ref. [20]. However, so far neither a functional dependence nor a physical interpretation has been given. The microscopic understanding of this highly promising effect for application in AFM spintronics is thus still lacking.

In order to provide a quantitative description of the influence of the hopping $t$ and the bare charge gap $\Delta$ onto the MBS, we plot $\Gamma_{\mathrm{MG}}(0) / t$ vs $t / \Delta$ in Fig. 4 for various combinations of $t$ and $\Delta$. This demonstrates clearly that the MBS in the Mott regime is proportional to the exchange coupling $J=4 t^{2} / \Delta$. Since the figure renders $\Gamma_{\mathrm{MG}}(0)$ in units of $t$, the proportionality $\Gamma_{\mathrm{MG}}(0) \propto J$ implies a straight line as depicted in red. It fits very well to the blue data for small values of $t / \Delta$ with a slope of 57.8 , which is equivalent to $\Gamma_{\mathrm{MG}}(0) \approx 14.4 \mathrm{~J}$ underlining its magnetic origin. Thus this effect is quite sizable and sets the scale for further contributions.

The MBS can also be linked to the decrease in the free energy when the system enters the MI phase. If such a decrease did not occur, the system would not display the phase transition to the ordered phase. The free-energy change below $T_{\mathrm{N}}$ is mainly due to the reduction of the internal energy, which can be determined solely from the single-particle spectral function. Upon transition from the PI to the MI, a redistribution of the weight within the spectral function occurs which leads to a large increase in the internal energy if it is not compensated by a MBS. This is exemplarily illustrated in Appendix B and corroborates that the MBS is a generic feature upon entering an antiferromagnetically ordered phase.

\section{HUBBARD-KONDO MODEL}

We extend the analysis presented so far for the Hubbard model to a model which includes localized spins so that it also bears features of a Kondo system. Specifically, we consider the Hubbard-Kondo model [42-44] given by

$$
\begin{aligned}
H_{\mathrm{HK}} & =H_{\mathrm{H}}+H_{\mathrm{K}}, \\
H_{\mathrm{K}} & =-2 J_{\mathrm{H}} \sum_{i} \vec{s}_{i} \cdot \vec{S}_{i},
\end{aligned}
$$

where $H_{\mathrm{H}}$ is the Hubbard model equation (1). The Kondo term $H_{\mathrm{K}}$ couples the spin of the electron $\vec{s}_{i}$ ferromagnetically to the local spin $\vec{S}_{i}$ originating from a Hund coupling. We choose the

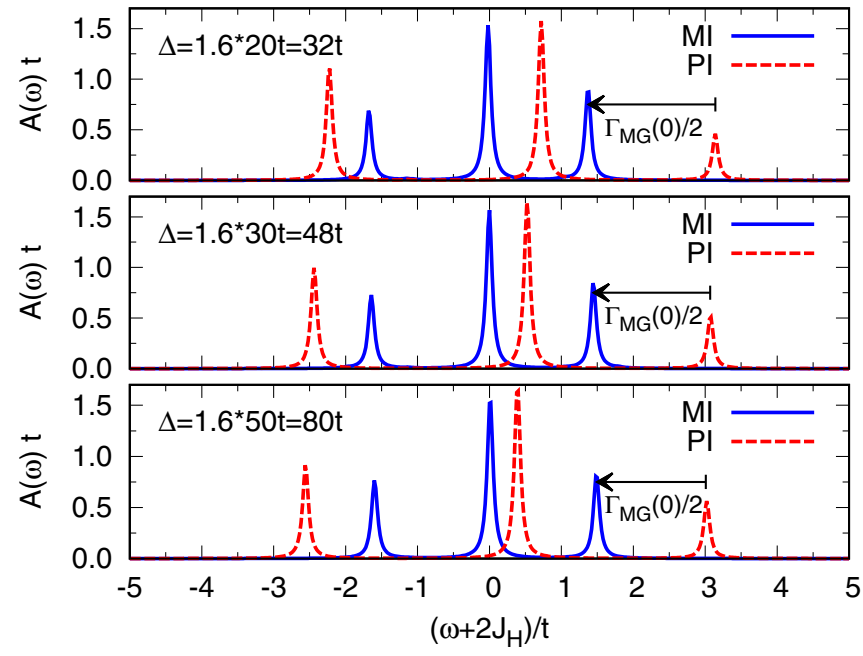

FIG. 5. The same as Fig. 3, but for the Hubbard-Kondo model with the local spin $S=2$ and the Hund coupling $J_{\mathrm{H}}=0.15 U$. The bare charge gap is given by $\Delta=U+4 J_{\mathrm{H}}=1.6 U$. The spectral functions do not approach each other upon increasing $U$.

local spin quantum number to be $S=2$ and the Hund coupling to be $J_{\mathrm{H}}=0.15 U$. The bare charge gap is no longer given by $U$ alone but acquires a contribution from the Hund coupling, $\Delta=U+4 J_{\mathrm{H}}$.

Figure 5 depicts the local spectral functions as in Fig. 3 but for the Hubbard-Kondo model. In contrast to data from the Hubbard model, Fig. 5 shows that the spectral functions in the PI and in the MI remain distinctly different even for large $\Delta$ resulting in an enhanced MBS of the Mott gap $\Gamma_{\mathrm{MG}}(0)$; see the indicated arrows. This can clearly be associated with the noticeably smaller bandwidth in the MI phase compared with the PI phase, which is the fingerprint of the double-exchange mechanism [45-48].

The double-exchange mechanism is well known for enhancing the mobility of an electron in a ferromagnetic state since the 1950s, and it is responsible for ferromagnetism in perovskite manganites. In an AFM state, however, the double-exchange mechanism strongly suppresses the effective hopping between sites with antiparallel spin ordering.

The basic idea of the mechanism is illustrated in Fig. 6. A hole added to the half-filled system propagates with an effective hopping which determines the bandwidth of the single-particle spectral function. In the limit of large $J_{\mathrm{H}}$ it is natural to restrict the local Hilbert space such that the electron spin $s=1 / 2$ and the local spin $S$ always form the maximum total spin $S+1 / 2$. This allows the following relation to be derived for the effective hopping between sites $i$ and $j$ [49]:

$$
\frac{t_{i, j}^{\text {eff }}}{t}=\frac{\mathcal{S}_{i, j}^{T}+1 / 2}{2 S+1}(-1)^{2 S-\mathcal{S}_{i, j}^{T}+1 / 2},
$$

where $\mathcal{S}_{i, j}^{T}$ is the total bond spin constructed from the spin at site $i$ and the spin at site $j$, i.e., from $S+1 / 2$ and $S$ [50]. One notes that there is always a hole either at site $i$ or at site $j$. The total bond spin takes the values $\mathcal{S}_{i, j}^{T}=$ $1 / 2,3 / 2, \ldots, 2 S+1 / 2$. For parallel spin ordering between sites $i$ and $j$ we have $\mathcal{S}_{i, j}^{T}=2 S+1 / 2$, which results in the effective hopping $t_{i, j}^{\mathrm{eff}}=t$. The PI phase is described by singlet 
(a) antiferromagnetic

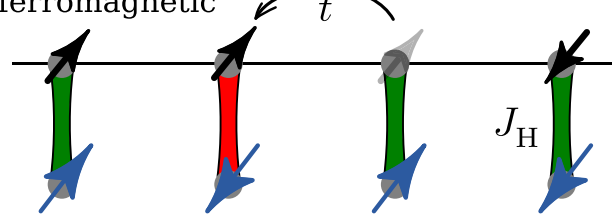

(b) ferromagnetic

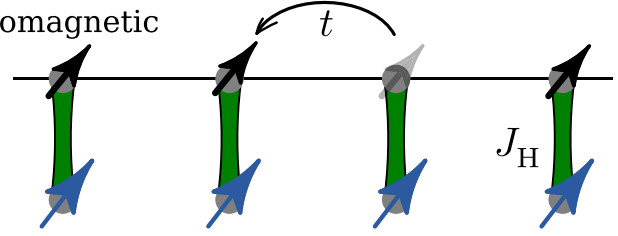

FIG. 6. Illustration of the reduction of the effective hopping due to the double-exchange mechanism. Due to the strong Hund coupling only electrons with spin aligned with the local spin can occur. This allows unrestricted hopping between sites with parallel spin orientation; see (b). However, for antiparallel spin orientation, no hopping is possible; see (a).

bonds, i.e., bonds with the total spin 0 . Adding a hole to a singlet bond creates a bond with the total spin $\mathcal{S}_{i, j}^{T}=1 / 2$, which can be realized from the commutation relation

$$
\left[\overrightarrow{\mathcal{S}}_{i, j}^{T} \cdot \overrightarrow{\mathcal{S}}_{i, j}^{T}, c_{j, \alpha}\right]=\frac{3}{4} c_{j, \alpha}-\sum_{\beta} c_{j, \beta} \vec{\sigma}_{\alpha, \beta} \cdot \overrightarrow{\mathcal{S}}_{i, j}^{T},
$$

where $\overrightarrow{\mathcal{S}}_{i, j}^{T}=\vec{s}_{i}+\vec{S}_{i}+\vec{s}_{j}+\vec{S}_{j}$ is the total bond spin operator and $\vec{\sigma}$ is a vector made of Pauli matrices. Such a hole propagates with the effective hopping $t_{i, j}^{\text {eff }}=t(-1)^{2 S} /(2 S+1)$ according to Eq. (3). In the case of antiparallel spin ordering between sites $i$ and $j$, a pure hopping can never take place, i.e., the hopping of the hole is always accompanied by the reduction of the local magnetic numbers from the absolute maximum values [49].

The above discussion explains the narrower bandwidth we observe for the Hubbard-Kondo model in Fig. 5 in contrast to the results for the Hubbard model in Fig. 3. One notes that the effective hopping in the Hubbard model for both MI and PI phases is the bare hopping $t$. More importantly, the above discussion explains the narrower bandwidth we observe in the MI phase in contrast to the PI phase in Fig. 5, which is the origin of the enhanced MBS in the Hubbard-Kondo model.

The results for the MBS in the Hubbard-Kondo model at $T=0$ in units of $t$ are included in Fig. 4. The qualitative behavior of the MBS in the Hubbard-Kondo model significantly differs from those of the Hubbard model. A substantial offset in the limit $t / \Delta \rightarrow 0$ is observed in the quantity $\Gamma_{\mathrm{MG}}(0) / t$. A linear fit given by the gray line

$$
\frac{\Gamma_{\mathrm{MG}}(0)}{t}=C_{1}+4 C_{2} \frac{t}{\Delta}
$$

with the constants $C_{1}=2.7$ and $C_{2}=6.3$ nicely agrees with our data. Note that the exchange coupling is given by $J=$ $4 t^{2} / \Delta$ such that we end with the fit

$$
\Gamma_{\mathrm{MG}}(0)=C_{1} t+C_{2} J .
$$

By plotting $\Gamma_{\mathrm{MG}}(0) / t$ vs $t / \Delta$ in Fig. 4 we can separate the two different contributions to the MBS more clearly: one proportional to the hopping $t$ which appears as a constant term and one proportional to the magnetic exchange $J$ which appears as a linear term. The first contribution results from changes in the effective hopping, and we refer to it as the hopping or the double-exchange contribution. The second contribution results from the alternating magnetic field as in the Hubbard model, and we refer to it as the exchange contribution.

Our findings unfold the essential role that the doubleexchange mechanism can play in the future development of AFM spintronics: It induces a coupling between the magnetic order and the charge gap as large as the hopping. The relation equation (6) is highly promising since there are several entire classes of compounds which show the exchange and the double-exchange effects. We leave a more detailed investigation of the hopping and the exchange contributions of the MBS to future research and instead apply our approach to a real material for the rest of this paper.

\section{APPLICATION TO $\alpha$-MnTe}

Now we apply the acquired understanding of the MBS in the Hubbard-Kondo model to a real compound: $\alpha$-MnTe. This AFM semiconductor displays a noticeable additional increase of the optical gap below its Néel temperature $T_{\mathrm{N}} \approx 310 \mathrm{~K}$. To separate the MBS from other temperature-dependent contributions, which are continuous, the experimental band gap is fitted in the paramagnetic regime $T>T_{\mathrm{N}}$ by the empirical Varshni function [51], which allows one to extrapolate the temperature dependence of the band gap in a paramagnetic semiconductor down to zero temperature. The difference between the actually measured gap and the extrapolated value quantifies the MBS [30,31]. Similar analyses were performed also for other magnetic semiconductors [27-29,52].

The magnetic order in $\alpha$-MnTe consists of planes of $\mathrm{Mn}^{2+}$ ions forming triangular lattices in which spins are parallel ordered. These planes are stacked, and the spins are oriented antiparallel in adjacent planes generating AFM order. According to Hund's rule the total spin at the $\mathrm{Mn}^{2+}$ ions is $S=5 / 2$ due to the half-filled $d$ shell. The dispersion of the collective magnons is well understood $[32,33,53]$. In contrast, the knowledge of the electronic excitations is significantly less developed, and the understanding of its coupling to the magnetic system is still in its infancy. Density functional theory (DFT) calculations $[33,54,55]$ indicate that the conduction band in $\alpha$-MnTe is dominated by Mn $3 d$ contributions although the Mn $4 s$ orbital is also involved. Assuming scenario (ii), we neglect the $4 s$ admixture and treat $\alpha$-MnTe as a charge-transfer insulator [16] where the optical gap $g$ arises from promoting an electron from the filled $p$ band of $\mathrm{Te}^{2-}$ to the empty upper Hubbard band $d^{+}$at $\mathrm{Mn}^{2+}$; cf. the panel for $T_{1}$ in Fig. 7 .

Figure 7 schematically depicts the relative change in bands upon lowering the temperature $T_{1} \rightarrow T_{2} \rightarrow T_{3}$. As the temperature is decreased from $T_{1}$ to $T_{2}>T_{\mathrm{N}}$ the $p$ and the $d^{+}$ bands shift apart with the Mott gap remaining unchanged. This increase in the charge-transfer gap $g$ is induced by slight structural changes and minute temperature effects in the paramagnetic phase. This fraction of the change in the gap is continuous through the magnetic transition and thus captured by the extrapolation with the Varshni function. For $T_{3}<T_{\mathrm{N}}$ an additional contribution $\Delta d^{+}$to the gap arises due to the MBS of the upper Hubbard band $d^{+}$. In principle, the magnetic 


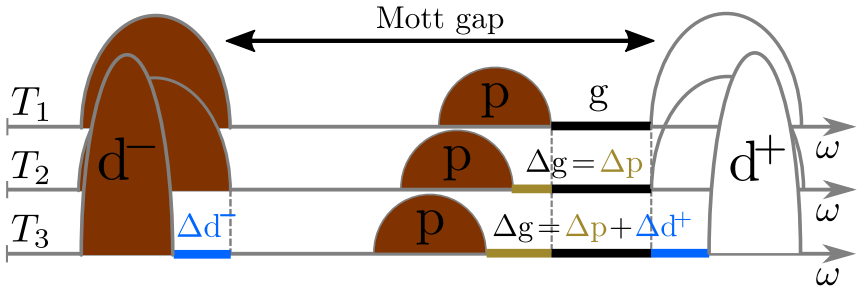

FIG. 7. Sketch of the bands in $\alpha$-MnTe comprising the $p$ bands at $\mathrm{Te}^{2-}$ and the lower $\left(d^{-}\right)$and upper $\left(d^{+}\right)$Hubbard bands of the $3 d$ electrons at $\mathrm{Mn}^{2+}$ at three temperatures $T_{1}>T_{2}>T_{\mathrm{N}}>T_{3}$, where $T_{\mathrm{N}}$ is the Néel temperature. The energy difference between the $d^{-}$ and the $d^{+}$Hubbard bands defines the Mott gap, and the energy difference between the $p$ and the $d^{+}$band defines the charge-transfer gap $g$. The optical gap equals the charge-transfer gap. The Mott gap experiences the magnetic blue shift $\Gamma_{\mathrm{MG}}\left(T_{3}\right)=\Delta d^{+}+\Delta d^{-}$, while the charge-transfer gap experiences the magnetic blue shift $\Gamma_{\mathrm{CTG}}\left(T_{3}\right)=\Delta d^{+} ;$see main text.

ordering could also affect the $p$ band; however, this would be an indirect effect, and we thus assume it to be less relevant. Consequently, to address the MBS in $\alpha$-MnTe, we focus on an effective Hamiltonian describing the electrons in the $3 d$ shell of the $\mathrm{Mn}^{2+}$ ions.

For a quantitative description, the established Heisenberg model for the spins of the $\mathrm{Mn}^{2+}$ ions [32,33] needs to be extended by the charge degrees of freedom. The full extension would require us to consider at least five $d$ bands from the $\mathrm{Mn}^{2+}$ ions plus three $p$ bands from the $\mathrm{Te}^{2-}$ ions. This is by far too complex for an explicit numerical treatment of the strong interactions present at the Mn sites. For this reason, we follow the idea proposed in Ref. [30] and describe the itineracy of each of the five $d$ electrons in a one-band Hubbard model while treating the other four $d$ electrons as localized forming a spin $S=2$. We stress that the itinerant electron is representative for all five electrons. We do not claim that the five orbitals are different, but that for each electron in one of them the other four act like a localized spin. In other words, we make the approximation that the local Fock space of the $\mathrm{Mn}^{+2} 3 d$ orbitals is restricted to the charge configurations $N=4,5$, and 6 , so that we only need to take into account the charge fluctuation in one effective local orbital which is degenerate with respect to spin. This is well justified since we are interested in the low-energy charge excitations, specifically, the charge gap.

Hence we consider a Hubbard-Kondo lattice model on stacked triangular lattices (cf. Fig. 8),

$$
\begin{aligned}
H= & -\sum_{i, j} \sum_{\sigma=\uparrow, \downarrow} t_{i, j}\left(c_{j, \sigma}^{\dagger} c_{i, \sigma}+\text { H.c. }\right)+U \sum_{i} n_{i, \downarrow} n_{i, \uparrow} \\
& -2 J_{\mathrm{H}} \sum_{i} \vec{S}_{i} \cdot \vec{s}_{i}+\sum_{i, j} J_{i, j}\left(\vec{S}_{i} \cdot \vec{s}_{j}+\vec{S}_{j} \cdot \vec{s}_{i}+\vec{S}_{i} \cdot \vec{S}_{j}\right),
\end{aligned}
$$

where the $t_{i, j}$ are the hopping elements and $J_{i, j}$ are the magnetic couplings; see Fig. 8(a). These effective magnetic couplings $J_{i, j}$ result from virtual excitations of the four $d$ orbitals, which are treated as local, to the neighboring Mn sites. (a)

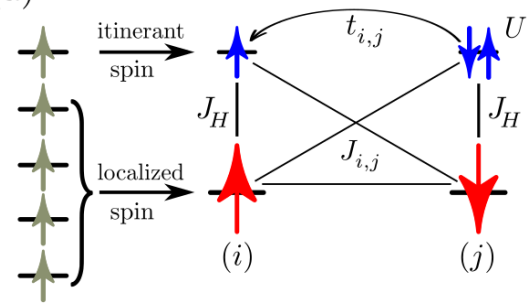

(b)

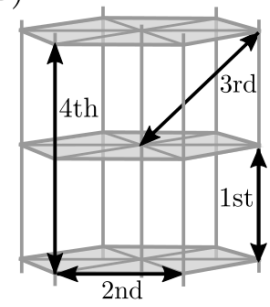

FIG. 8. (a) Illustration of the Hubbard-Kondo model (7) for the half-filled $3 d$ shell of $\mathrm{Mn}^{2+}$ ions at two sites $i$ and $j$. (b) Stacked triangular layers with first, second, third, and fourth neighbors specified so that we distinguish $t_{1}, t_{2}, t_{3}, t_{4}$ and $J_{1}, J_{2}, J_{3}, J_{4}$.

The intersite couplings are limited to the four nearest neighbors specified in Fig. 8(b). We denote the hopping and the magnetic coupling of the $n$th neighbor by $t_{n}$ and $J_{n}$. The magnetic couplings are taken from the measured magnon dispersion [33] to be $J_{1}=3.072 \mathrm{meV}, J_{2}=0.0272 \mathrm{meV}$, $J_{3}=0.4 \mathrm{meV}$, and $J_{4}=0.16 \mathrm{meV}$, matching also the observed Néel temperature. The Hubbard interaction $U$ ranges between $\approx 5 \mathrm{eV}$ and $\approx 7 \mathrm{eV}$, and the Hund coupling ranges between $\approx 0.7 \mathrm{eV}$ and $\approx 1.0 \mathrm{eV}$, based on estimates from atomic physics [30] and the DFT [33,54] calculations. We investigate the effect of $U$ and $J_{\mathrm{H}}$ on the MBS in this parameter regime. The hopping elements $t_{n}$ are determined such that they are consistent with the intersite exchange couplings, i.e., $J_{n}=4 t_{n}^{2} / \Delta$, where $\Delta$ is the bare charge gap $U+4 J_{\mathrm{H}}$. This is to guarantee that the low-energy spin excitations of the Hamiltonian equation (7) are described by the $S=5 / 2$ Heisenberg model already established for $\alpha$-MnTe by inelastic neutron scattering measurements [33]. The explicit values of the parameters are given in Appendix C. It must be noted that the larger, dominant hoppings $t_{1}$ and $t_{3}$ link sites with AFM ordering. Hence we expect a noticeable hopping contribution to the MBS to occur, stemming from the double-exchange mechanism described in Sec. III.

The DMFT accurately accounts for the local interactions $U$ and $J_{\mathrm{H}}$. In the limit of infinite coordination number justifying DMFT, the intersite interactions $J_{n}$ are consistently treated by static mean fields [56]. Thus the intersite magnetic interactions are represented by

$$
H_{\mathrm{MF}}=-\sum_{i}\left(h_{i}^{\mathrm{loc}} S_{i}^{z}+h_{i}^{\mathrm{iti}} s_{i}^{z}\right),
$$

where the effective magnetic fields

$$
\begin{aligned}
h_{i}^{\mathrm{loc}} & =2\left(J_{1}-3 J_{2}+6 J_{3}-J_{4}\right)\left\langle S_{i}^{z}+s_{i}^{z}\right\rangle, \\
h_{i}^{\mathrm{iti}} & =2\left(J_{1}-3 J_{2}+6 J_{3}-J_{4}\right)\left\langle S_{i}^{z}\right\rangle
\end{aligned}
$$

act on the localized spin $\left(h_{i}^{\text {loc }}\right)$ and on the itinerant spin $\left(h_{i}^{\mathrm{iti}}\right)$, respectively. They need to be determined self-consistently. For simplicity, we take the magnetization in the $z$ direction although a weak spin-orbit coupling orients it in the $x$ direction $[33,55]$. However, for the spin-isotropic model studied here this does not matter.

The resulting Hamiltonian is solved using DMFT starting from an initial guess for the self-energy and the local magnetizations $\left\langle S_{i}^{z}\right\rangle$ and $\left\langle s_{i}^{z}\right\rangle$. These quantities are updated in 

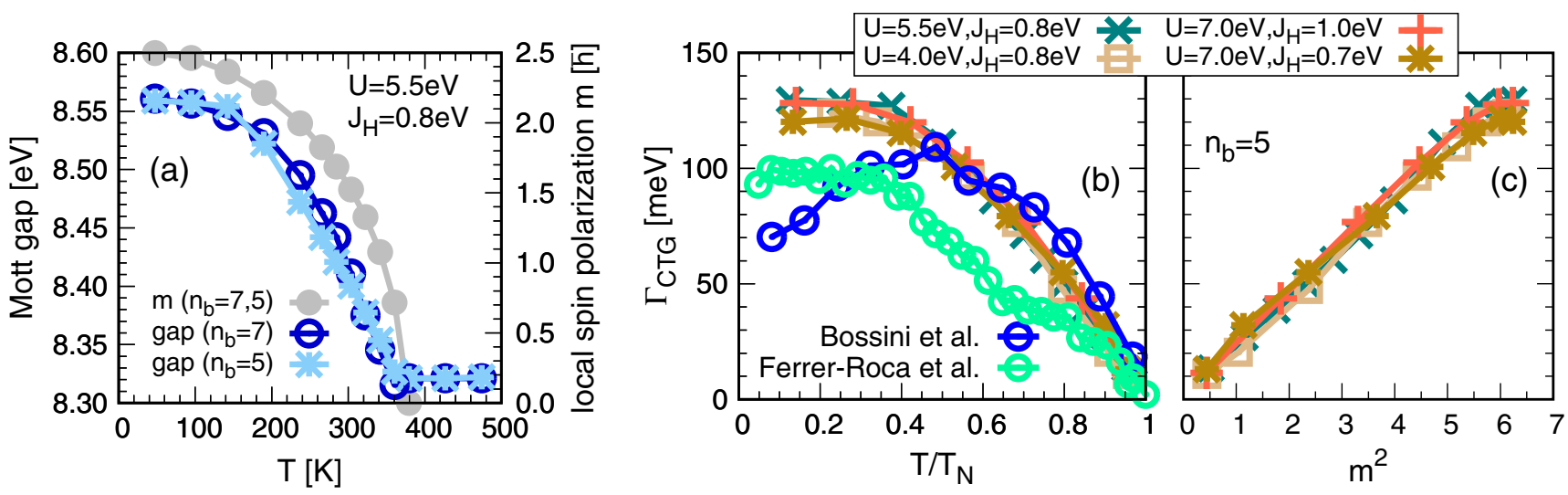

FIG. 9. (a) Theoretical results for the Mott gap and the local spin polarization $m$ vs $T$. (b) Theoretical and experimental (given by Bossini et al. [30] and Ferrer-Roca et al. [31]) results for the MBS in $\alpha$-MnTe as a function of temperature. (c) MBS vs the squared spin polarization $m^{2}$ combining the theoretical results from (a) and (b) for various values of $U$ and $J_{\mathrm{H}}$ for $n_{b}=5$.

each DMFT loop until convergence is reached within some tolerance; see Appendix A 2. This approach is well justified and goes far beyond the previous two-site calculation [30] because it properly treats the extended lattice and the dynamics of single charges and it allows us to study the temperature dependence. Of course, more sophisticated calculations are conceivable in the future to fix numerical values to higher accuracy $[20,57,58]$, but our aim here is to elucidate the fundamental physics.

In Fig. 9(a) we plot the temperature dependence of the Mott gap as obtained with $n_{b}=5$ and $n_{b}=7$ bath sites for $U=5.5 \mathrm{eV}$ and $J_{\mathrm{H}}=0.8 \mathrm{eV}$. The agreement of both data sets underlines that the results do not depend significantly on the number of bath sites. In addition, the local spin polarization $m=\left|\left\langle S_{i}^{z}+s_{i}^{z}\right\rangle\right|$ is shown, coinciding for $n_{b}=5$ and $n_{b}=7$ and indicating a Néel temperature $T_{\mathrm{N}} \approx 380 \mathrm{~K}$. This value represents a classical estimate since the DMFT approach does not capture intersite fluctuations, which are shown [33] to reduce $T_{\mathrm{N}}$ to $\approx 310 \mathrm{~K}$ in accordance with experiment $[53,59,60]$. Hence the effect of the neglected intersite fluctuations on the gap appears to be about $6 \mathrm{meV}(\approx 70 \mathrm{~K})$.

The Mott gap remains almost independent of temperature in the paramagnetic phase $T \geqslant 380 \mathrm{~K}$ in line with our findings in the Hubbard model. This result supports the assumption in Fig. 7 that for $T>T_{\mathrm{N}}$ the Mott gap remains unchanged and the increase in the charge-transfer gap $g$ is essentially due to a smooth relative shift of the $p$ band captured by the Varshni fit. The antiferromagnetic ordering induces a MBS of the Mott gap $\Gamma_{\mathrm{MG}}(T)$ of approximately $250 \mathrm{meV}$ as $T \rightarrow 0$. This is the shift between the lower $d^{-}$and the upper $d^{+}$Hubbard bands in Fig. 7, i.e., $\Delta d^{+}+\Delta d^{-}$. The MBS of the charge-transfer gap $\Gamma_{\mathrm{CTG}}(T)$, which is the MBS measured in the experiment, is given by $\Delta d^{+}$. Since we cannot calculate the individual contributions $\Delta d^{ \pm}$separately, we assume that they are shifted symmetrically, i.e., $\Delta d^{+}=\Delta d^{-}$, typical for a half-filled Mott insulator. This implies that the theoretical MBS of the chargetransfer gap $\Gamma_{\mathrm{CTG}}(T)=\Gamma_{\mathrm{MG}}(T) / 2$ is about $120 \mathrm{meV}$ at its maximum.

Figure 9(b) shows $\Gamma_{\text {СTG }}$ as function of $T / T_{\mathrm{N}}$. For all four pairs of $U$ and $J_{\mathrm{H}}$ we find the same Néel temperature $T_{\mathrm{N}} \approx$ $380 \mathrm{~K}$, which is to be expected since $T_{\mathrm{N}}$ is determined from the low-energy Heisenberg model defined by the intersite exchange couplings $J_{n}$, which we kept fixed; for tables of the explicit parameters used, see Appendix C. However, the Mott gap changes significantly from $\approx 7 \mathrm{eV}$ for $U=4.0 \mathrm{eV}$ and $J_{\mathrm{H}}=0.8 \mathrm{eV}$ to $\approx 11 \mathrm{eV}$ for $U=7.0 \mathrm{eV}$ and $J_{\mathrm{H}}=1.0 \mathrm{eV}$. Remarkably, there is hardly any change in the MBS despite this large change in the Mott gap. This corroborates that the essential parameters for the MBS are the exchange couplings and the hopping elements as indicated by Eq. (6) above.

We also added the experimental results for the MBS to Fig. 9(b). The theoretical data agree nicely with the data of Bossini et al. [30] for $T \gtrsim 0.5 T_{\mathrm{N}}$. It is mentioned by FerrerRoca et al. [31] that their data probably underestimate the MBS between $T \approx 0.45 T_{\mathrm{N}}$ and $T \approx 0.65 T_{\mathrm{N}}$. The results of Bossini et al. deviate from theory below $T \approx 0.5 T_{\mathrm{N}}$, where the experimental data turn down in contrast to expectation and the data set from Ref. [31]. The deviating downturn is likely due to experimental reasons, e.g., sample quality and/or stability of the experimental conditions. In fact, due to the saturation of the spin polarization at low temperatures we expect the MBS also to saturate as is found by Ferrer-Roca $e t$ al. for $T<0.4 T_{\mathrm{N}}$. We emphasize that the very good agreement between experiment and theory in Fig. 9(b) is achieved using generic parameters from the literature for the Hubbard-Kondo model without any fine-tuning in contrast to the approach in Ref. [29]. This provides strong evidence that the observed blue shift upon ordering is the generic MBS of the advocated Hubbard-Kondo lattice model.

Finally, Fig. 9(c) combines the calculated $\Gamma_{\mathrm{CTG}}(T)$ and the local spin polarization $m(T)$ eliminating the temperature. The value of the gap at $T_{\mathrm{N}}$ is fixed such that the MBS vanishes for $m^{2} \rightarrow 0$. The figure clearly shows that there is an almost linear relation between the squared local spin polarization $m^{2}$ and the MBS, $\Gamma_{\mathrm{CTG}}(T) \propto m^{2}(T)$, for various parameter combinations of the local interactions $U$ and $J_{\mathrm{H}}$. This behavior is in line with previous experimental findings [31] and underlines that the MBS is a robust effect not depending on details. Reference [29] also finds a MBS $\propto m^{2}$, but the computation strongly depends on the chosen parameters since it originates from scenario (i), in which the magnetic order affects the itinerant electrons only indirectly.

The MBS in Hubbard-Kondo models is governed by a contribution from the hopping and a contribution from the 


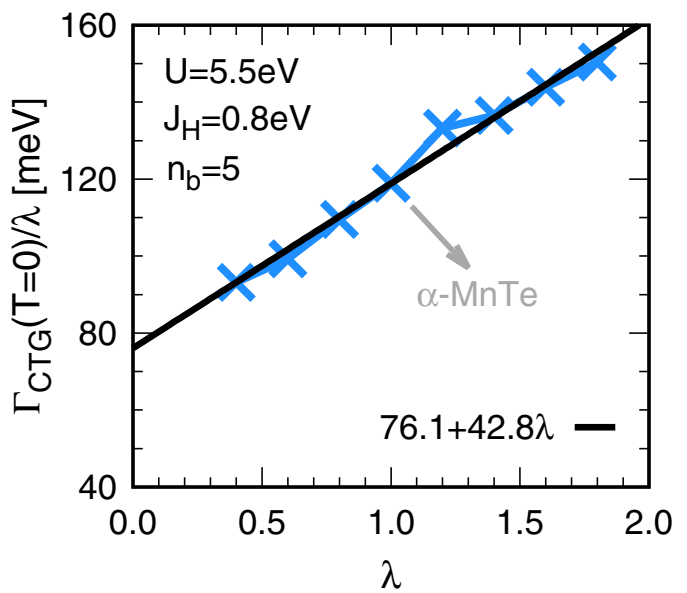

FIG. 10. MBS at $T=0$ as a function of the scaling parameter of the hopping $\lambda$ as given in Eqs. (10a) and (10b). As in Fig. 4, the MBS offset on the $y$ axis is proportional to the hopping stemming from the double-exchange mechanism, while the slope results from the contribution to the MBS proportional to the magnetic exchange couplings. Note that for $\alpha$-MnTe $(\lambda=1)$ the hopping contribution equals roughly twice the exchange contribution.

intersite exchange interaction; see Eq. (6). We investigate this point for $\alpha$-MnTe as well by means of a plot analogous to Fig. 4. In view of the numerous parameters relevant for $\alpha$ MnTe (four hopping elements, four exchange couplings, the Hubbard interaction, and the Hund coupling) a variation of individual parameters appears to be not practical. Hence we resort to a uniform scaling by a parameter $\lambda$ according to

$$
\begin{aligned}
t_{n} & \rightarrow t_{n}(\lambda)=\lambda t_{n} \\
\Rightarrow \quad J_{n} & \rightarrow J_{n}(\lambda)=\lambda^{2} J_{n}
\end{aligned}
$$

leaving the local interactions $U$ and $J_{H}$ unchanged. The plot $\Gamma_{\mathrm{CTG}}(T=0) / \lambda$ vs $\lambda$ for $U=5.5 \mathrm{eV}$ and $J_{\mathrm{H}}=0.8 \mathrm{eV}$ in Fig. 10 recreates the same kind of analytical dependence as Fig. 4. Note that for $\lambda<2$ we have $\lambda t_{n} / \Delta<0.02$, which corresponds to the deep Mott regime. As expected, we find the same qualitative behavior as in Fig. 4 described very well by a linear fit. The offset at $\lambda=0$ is the contribution proportional to the hoppings due to the rescaled $\Gamma_{\mathrm{CTG}}(0) / \lambda$ plotted. In the same way, the slope results from contributions proportional to the magnetic exchange couplings $J_{n}(\lambda) \propto \lambda^{2}$. The plot in Fig. 10 again allows us to separate the two contributions, as the plot in Fig. 4 did for the Hubbard-Kondo model. The nice linear behavior suggests a hopping contribution of about $76 \mathrm{meV}$ and an exchange contribution of about $43 \mathrm{meV}$ to the MBS in $\alpha$-MnTe. This large hopping contribution emphasizes the important role of the double-exchange mechanism in the MBS in systems with localized spins.

\section{CONCLUSIONS}

We established that the MBS in the Mott regime of the three-dimensional (3D) Hubbard model stems from the magnetic exchange coupling. While the decrease in the MBS with increasing interaction $U$ has been observed before [20], its proportionality to the magnetic exchange $J=4 t^{2} / U$ is a finding which sets the energy scale for further contributions.

Our key result relates to systems which involve localized spins in addition to itinerant electrons. For the HubbardKondo model with a ferromagnetic Kondo coupling we showed that there are two contributions to the MBS: one similar to the MBS in the Hubbard model which is proportional to the magnetic exchange, and another which is proportional to the hopping. The latter stems from the double-exchange mechanism, which reduces the effective hopping between sites with antiparallel spin ordering.

This finding opens up a route to applications of the MBS since a plethora of heavily investigated systems consist of itinerant electrons and localized spins, for instance, the manganites. Exemplarily, we elucidated the origin of the experimentally established MBS in $\alpha$-MnTe, which is a promising candidate for applications with AFM order at room temperature. We developed an extended Hubbard-Kondo lattice model for $\alpha$-MnTe. The MBS found in this model is in overall excellent agreement with the experimental findings for $\alpha$-MnTe.

Strong MBSs in magnetic semiconductors can play a major role in spin-to-charge conversion on the femtosecond time scale, which is the characteristic time scale of the hopping and the intersite exchange interactions. Recent progress in the manipulation of magnons in antiferromagnets on ultrafast time scales $[24,61]$ adds to the relevance of a comprehensive understanding of the coupling of spin and charge dynamics [62]. A major outlook of our work consists in exploring the role of the dimensionality of a Mott system in the MBS. This is highly relevant in view of both the massive present research activity focused on 2D materials and the widely explored properties of low-dimensional magnetic semiconductors. Hence the demonstrated MBS paves a promising route for future research, both fundamental and applied.

\section{ACKNOWLEDGMENTS}

This study was funded by the German Research Foundation (DFG) and the Russian Foundation for Basic Research in the International Collaborative Research Centre TRR 160 (Project B8) and by DFG Grant No. BO 5074/1-1.

\section{APPENDIX A: THEORETICAL APPROACH}

\section{General remarks}

We use dynamical mean-field theory (DMFT) [3], which is an established approach for strong local interactions and large coordination number. The frequency-dependent self-energy allows us to describe paramagnetic Mott insulators, not accessible by static mean-field theories. We use the real-space DMFT (RDMFT) method [63-65] as implemented by one of us [66] and applied successfully to various models [67-69]. We note that for the bulk properties it is not necessary to use the real-space extension of DMFT. However, in view of future analysis of the spatial dependence in thin films as in Ref. [30] we opt for RDMFT for comparability.

Exact diagonalization (ED) is employed as the impurity solver [41] providing direct access to dynamics at real frequencies and the quantum mechanical treatment of localized 
spins going beyond previous classical approximations based on the quantum Monte Carlo solver [44,70,71]. The local spectral function $A(\omega)$ results from the imaginary part of the local Green's function, averaged over both sublattice sites. We compute the Mott gap from the positions of the peaks in the spectral function. Although the spectral function for a finite number of bath sites $n_{b}$ consists of a series of sharp peaks approximating the continuous function, the Mott gap is found to be accurate and is used to benchmark the results of other methods [19]. We use the chemical potential $\mu$ to satisfy the condition of half filling. The lattice system is approximated by clusters of $L \times L \times L$ sites with $L=10$. We checked for selected temperatures close to the transition temperature that the results remain the same for $L=20$.

\section{Dynamical mean-field theory of the Hubbard-Kondo model}

After the mean-field decoupling of the intersite magnetic interactions shown in (8) the Hamiltonian from (7) reads

$$
\begin{aligned}
H= & -\sum_{i, j} \sum_{\sigma} t_{j, i}\left(c_{j, \sigma}^{\dagger} c_{i, \sigma}+\text { H.c. }\right)-\sum_{i}\left(h_{i}^{\mathrm{iti}} s_{i}^{z}+\mu n_{i}\right) \\
& +U \sum_{i} n_{i, \downarrow} n_{i, \uparrow}-2 J_{H} \sum_{i} \vec{S}_{i} \cdot \vec{s}_{i}-\sum_{i} h_{i}^{\mathrm{loc}} S_{i}^{z}
\end{aligned}
$$

with the effective magnetic fields

$$
\begin{aligned}
h_{i}^{\mathrm{loc}} & =2\left(J_{1}-3 J_{2}+6 J_{3}-J_{4}\right)\left\langle S_{i}^{z}+s_{i}^{z}\right\rangle, \\
h_{i}^{\mathrm{iti}} & =2\left(J_{1}-3 J_{2}+6 J_{3}-J_{4}\right)\left\langle S_{i}^{z}\right\rangle
\end{aligned}
$$

acting on the localized spin $\vec{S}_{i}$ and on the spin of the itinerant electrons $\vec{s}_{i}$, respectively, at the lattice site $i$. We added a chemical potential term $\mu$ to the Hamiltonian (A1) to control the electron density $n_{i}:=n_{i, \downarrow}+n_{i, \uparrow}$ in the system keeping it at half filling. In the derivation of Eqs. (A2a) and (A2b) we consider ferromagnetic order within the triangular layers and antiferromagnetic order between them. For simplicity, the magnetic order is taken to be in the $\hat{z}$ direction in spin space, but the choice of direction does not matter since we consider a fully spin isotropic model. The treatment of the weak anisotropy stemming from a spin-orbit coupling [33,55] is left to future research. The effective magnetic fields in Eqs. (A2a) and (A2b) depend on the local spin polarizations $\left\langle S_{i}^{z}\right\rangle$ and $\left\langle s_{i}^{z}\right\rangle$ and need to be determined self-consistently in the course of the iterations of the RDMFT.

Essentially, we use the RDMFT implementation of Ref. [66] for SU(2) systems with a generalization of the Anderson impurity model to an Anderson-Kondo impurity model which includes the additional local degrees of freedom, here the localized spin in Eq. (A1). Note that we treat the spin fully quantum mechanically. We also updated the implementation of Ref. [66] such that some local expectation values are computed during the RDMFT loop so that the mean fields can be modified iteratively. In the case of the Hamiltonian (A1) these local expectation values are the spin polarizations $\left\langle s_{i}^{z}\right\rangle$ and $\left\langle S_{i}^{z}\right\rangle$, needed for the calculations of the effective magnetic fields in Eqs. (A2a) and (A2b). We stress that the local Green's function, the self-energy, and the dynamical Weiss field are all diagonal in spin space as the Hamiltonian Eq. (A1) is diagonal in $S^{z}$. This simplifies the general formalism of Ref. [66].
The terms in the first line of Eq. (A1) describe the noninteracting parts of the itinerant electrons from which the noninteracting lattice Green's function is constructed. The second line in Eq. (A1) contains the Hubbard interaction between the itinerant electrons, the Hund coupling between the spin of the itinerant electron and the localized $\operatorname{spin} S=2$, and the effective magnetic field at the localized spin. They enter the calculation in the local impurity problem. The RDMFT loop starts with an initial guess for the self-energy matrix $\boldsymbol{\Sigma}\left(i \omega_{n}\right)$ and the local spin polarizations $\left\langle s_{i}^{z}\right\rangle$ and $\left\langle S_{i}^{z}\right\rangle$. The real-space lattice Green's function is calculated according to Dyson's equation

$$
\boldsymbol{G}\left(i \omega_{n}\right)=\left[i \omega_{n} \mathbb{1}-\boldsymbol{H}_{0}-\boldsymbol{\Sigma}\left(i \omega_{n}\right)\right]^{-1},
$$

where $\boldsymbol{H}_{0}$ is the matrix representation of the noninteracting terms in the first line of Eq. (A1). To address the local problem at the lattice site $i$, we use the Anderson-Kondo impurity model [72]

$$
\begin{aligned}
H_{i}= & -\mu n_{i}-h_{i}^{\mathrm{iti}} s_{i}^{z}+U n_{i, \downarrow} n_{i, \uparrow}-h_{i}^{\mathrm{loc}} S_{i}^{z}-2 J_{H} \vec{S}_{i} \cdot \vec{s}_{i} \\
& +\sum_{\ell=1}^{n_{b}} \sum_{\sigma} \epsilon_{\ell}^{i} a_{\ell, \sigma}^{\dagger} a_{\ell, \sigma}+\sum_{\ell=1}^{n_{b}} \sum_{\sigma}\left(a_{\ell, \sigma}^{\dagger} V_{\ell, \sigma}^{i} c_{i, \sigma}+\text { H.c. }\right),
\end{aligned}
$$

where $a_{\ell, \sigma}^{\dagger}$ and $a_{\ell, \sigma}$ are the fermionic creation and annihilation operators at the bath site $\ell$ with the spin $\sigma=\uparrow, \downarrow$. The bath sites in Eq. (A4) approximate the effect of the surrounding sites in the lattice [3]. The bath parameters $\epsilon_{\ell}^{i}$ and $V_{\ell, \sigma}^{i}$ are determined by fitting the dynamical Weiss field $[41,66]$. The self-energy as well as the local spin polarizations $\left\langle s_{i}^{z}\right\rangle$ and $\left\langle S_{i}^{z}\right\rangle$ are calculated using ED of the Anderson-Kondo impurity model (A4). These quantities are employed for the next RDMFT iteration loop.

Since the model is symmetric with respect to a combined swap of the sublattice and the spin orientations, we only need to set up the impurity model (A4) for one representative site. In this sense, the lattice solutions are homogeneous. Hence one does not need to fully invert the matrix in Eq. (A3) because only the two columns for the two spin orientations at the representative site are needed [66]. This enables us to treat very large system sizes in Eq. (A3) so that finite-size corrections are completely negligible.

\section{APPENDIX B: INTERNAL ENERGY AND MAGNETIC BLUE SHIFT}

The internal energy of a general interacting fermionic system described by the Hamiltonian

$$
H=H_{0}+W=\sum_{i, j} h_{i, j} c_{i}^{\dagger} c_{j}+\frac{1}{2} \sum_{i, j, k, l} W_{i, j, k, l} c_{i}^{\dagger} c_{j}^{\dagger} c_{k} c_{l}
$$

can be expressed as [73]

$$
E:=\langle H\rangle=\frac{1}{2} \sum_{i, j} \int_{-\infty}^{+\infty} d \omega A_{i, j}(\omega) f(\omega)\left[\omega \delta_{i, j}+h_{i, j}\right],
$$

where $i$ and $j$ specify single-particle quantum numbers, $A_{i, j}(\omega)$ is the spectral function of the single-particle Green's function, and $f(\omega)$ is Fermi's occupation function. Equation 


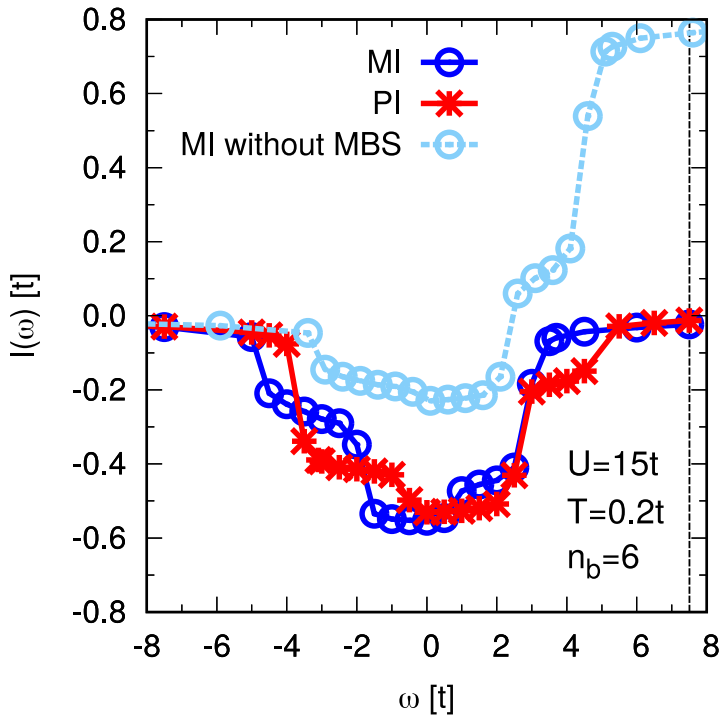

FIG. 11. The partial energy $I(\omega)$ defined in Eq. (B4) vs frequency $\omega$ changing over the lower Hubbard band for the 3D Hubbard model for $U=15 t$ and $T=0.2 t$ in the MI (dark blue) and in the PI (red) as well as in the MI without MBS (light blue). The vertical dashed line at $U / 2=7.5 t$ shows the Fermi energy. The number of bath sites is $n_{b}=6$ in the ED impurity solver.

(B2) shows that the internal energy can be determined solely from the single-particle spectral function. The first contribution in Eq. (B2) describes $\left\langle H_{0}\right\rangle / 2+\langle W\rangle$, while the second contribution equals half the kinetic energy, $\left\langle H_{0}\right\rangle / 2$.

The Mott gap separating the lower and the upper Hubbard bands is typically much larger than the Néel temperature $T_{\mathrm{N}}$. For temperatures $T \lesssim T_{\mathrm{N}}$ this essentially restricts the integration in Eq. (B2) to only the lower Hubbard band (LHB). Then, the first contribution in Eq. (B2) can be simplified to

$$
\varepsilon_{1}=\frac{E_{1}}{N}=\int_{\mathrm{LHB}} \omega A(\omega) d \omega,
$$

where $N$ is the number of lattice sites and we used the translational symmetry of the spin-averaged local spectral function $A(\omega)$, which we plotted in Fig. 1 .

In order to see how the energy in Eq. (B3) is distributed over frequency, we consider the partial energy

$$
I(\omega)=\int_{-\infty}^{\omega} \omega^{\prime} A\left(\omega^{\prime}\right) d \omega^{\prime},
$$

which equals $\varepsilon_{1}$ if $\omega$ is large enough to cover the whole LHB. In Fig. 11 we plot $I(\omega)$ for the 3D Hubbard model at $U=15 t$ and $T=0.2 t$ in the MI and in the PI as well as in the MI without any MBS of the local spectral function. The results are obtained using $n_{b}=6$ bath sites in the ED impurity solver. Figure 11 clearly shows that upon entering the magnetically ordered phase from the paramagnetic phase, a redistribution of the weight within the spectral function occurs which leads to a large increase in the internal energy if it is not compensated by a MBS. Such a redistribution has been observed also in Ref. [18], both experimentally and theoretically.

For the Hubbard model $H=H_{t}+H_{U}$ with the nearestneighbor hopping term $H_{t}$ and the Hubbard interaction $H_{U}$ we

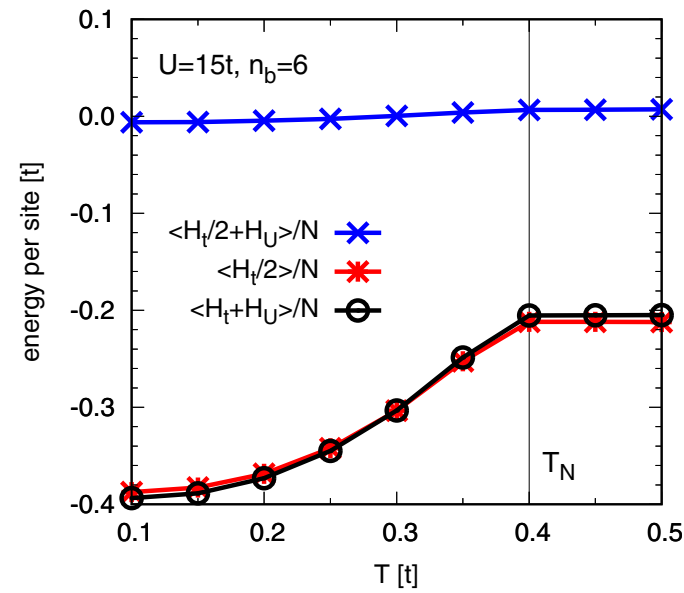

FIG. 12. The internal energy $\left\langle H_{t}+H_{U}\right\rangle$ and the individual contributions $\left\langle H_{t} / 2+H_{U}\right\rangle$ and $\left\langle H_{t} / 2\right\rangle$ of the Hubbard model $H=H_{t}+$ $H_{U}$ per lattice site vs the temperature $T$ for the Hubbard interaction $U=15 t$ and $n_{b}=6$ bath sites in the ED impurity solver.

plot the internal energy $\left\langle H_{t}+H_{U}\right\rangle$ per lattice site in Fig. 12 for $U=15 t$ and $n_{b}=6$. We include also $\left\langle H_{t} / 2+H_{U}\right\rangle$ and $\left\langle H_{t} / 2\right\rangle$, corresponding to the first and the second contribution in Eq. (B2), respectively. We see that $\left\langle H_{t} / 2+H_{U}\right\rangle$ remains close to zero and the reduction of the internal energy below $T_{\mathrm{N}}$ is mainly due to $\left\langle H_{t} / 2\right\rangle$. Without MBS the contribution $\left\langle H_{t} / 2+H_{U}\right\rangle$ would increase substantially in the MI phase; see Fig. 11. This shows that the MBS is crucial to achieve a decrease in the internal energy, which is the prerequisite for the phase transition into the ordered phase to occur.

\section{APPENDIX C: MODEL PARAMETERS FOR $\alpha$-MnTe}

We fixed the intersite exchange interactions in $\alpha-\mathrm{MnTe}$ according to the values from Ref. [33]: $J_{1}=3.072 \mathrm{meV}, J_{2}=$ $0.0272 \mathrm{meV}, J_{3}=0.4 \mathrm{meV}$, and $J_{4}=0.16 \mathrm{meV}$. The hopping parameters corresponding to the different sets of the Hubbard interaction $U$ and the Hund coupling $J_{\mathrm{H}}$ are calculated from the relation

$$
J_{n}=4 t_{n}^{2} / \Delta
$$

with $\Delta=U+4 J_{\mathrm{H}}$. They are given in Table I for future convenient use.

TABLE I. The hopping parameters $t_{n}$ in $\alpha$-MnTe according to Eq. (C1) for the various sets of Hubbard interaction $U$ and Hund coupling $J_{\mathrm{H}}$. The hopping parameters are in units of $\mathrm{meV}$, and $U$ and $J_{\mathrm{H}}$ are in units of eV. The subscripts of the hopping parameters refer to the numbers in Fig. 8(b).

\begin{tabular}{lcccc}
\hline \hline$\left(U, J_{\mathrm{H}}\right)(\mathrm{eV})$ & $t_{1}(\mathrm{meV})$ & $t_{2}(\mathrm{meV})$ & $t_{3}(\mathrm{meV})$ & $t_{4}(\mathrm{meV})$ \\
\hline$(7.0,1.0)$ & 91.91 & 8.649 & 33.16 & 20.97 \\
$(7.0,0.7)$ & 86.75 & 8.163 & 31.30 & 19.80 \\
$(5.5,0.8)$ & 81.74 & 7.692 & 29.49 & 18.65 \\
$(4.0,0.8)$ & 74.36 & 6.997 & 26.83 & 16.97 \\
\hline \hline
\end{tabular}


TABLE II. The intersite exchange couplings $J_{n}$, the Hubbard interaction $U$, and the Hund coupling $J_{\mathrm{H}}$ in units of $t_{1}$. The rows correspond to the different parameter sets used in Table I.

\begin{tabular}{lcccc}
\hline \hline$\left(U, J_{\mathrm{H}}\right)$ & $J_{1}\left(10^{-2}\right)$ & $J_{2}\left(10^{-2}\right)$ & $J_{3}\left(10^{-2}\right)$ & $J_{4}\left(10^{-2}\right)$ \\
\hline$(76.16,10.88)$ & 3.342 & 0.02959 & 0.4352 & 0.1741 \\
$(80.69,8.07)$ & 3.541 & 0.03135 & 0.4611 & 0.1844 \\
$(67.29,9.787)$ & 3.758 & 0.03328 & 0.4894 & 0.1957 \\
$(53.79,10.76)$ & 4.131 & 0.03658 & 0.5379 & 0.2152 \\
\hline \hline
\end{tabular}

Expressing energies in units of $t_{1}$, as used in the DMFT calculations, one has the hopping parameters

$$
t_{n}=t_{1} \sqrt{J_{n} / J_{1}}
$$

independent of the choice of $U$ and $J_{\mathrm{H}}$. They are given by $t_{2}=0.0941 t_{1}, t_{3}=0.3608 t_{1}$, and $t_{4}=0.2282 t_{1}$. However, the intersite exchange couplings $J_{n}$ in units of $t_{1}$ depend on $U$ and $J_{\mathrm{H}}$. Table II provides $J_{n}, U$, and $J_{\mathrm{H}}$ in units of $t_{1}$ corresponding to the different parameter sets used in Table I.
[1] J. H. de Boer and E. J. W. Verwey, Semi-conductors with partially and with completely filled ${ }_{3} d$-lattice bands, Proc. Phys. Soc., London 49, 59 (1937).

[2] N. F. Mott, The basis of the electron theory of metals, with special reference to the transition metals, Proc. Phys. Soc., London, Sect. A 62, 416 (1949).

[3] A. Georges, G. Kotliar, W. Krauth, and M. J. Rozenberg, Dynamical mean-field theory of strongly correlated fermion systems and the limit of infinite dimensions, Rev. Mod. Phys. 68, 13 (1996).

[4] M. Imada, A. Fujimori, and Y. Tokura, Metal-insulator transitions, Rev. Mod. Phys. 70, 1039 (1998).

[5] C. Giannetti, M. Capone, D. Fausti, M. Fabrizio, F. Parmigiani, and D. Mihailovic, Ultrafast optical spectroscopy of strongly correlated materials and high-temperature superconductors: A non-equilibrium approach, Adv. Phys. 65, 58 (2016).

[6] P. A. Lee, N. Nagaosa, and X.-G. Wen, Doping a Mott insulator: Physics of high-temperature superconductivity, Rev. Mod. Phys. 78, 17 (2006).

[7] E. Manousakis, The spin- $\frac{1}{2}$ Heisenberg antiferromagnet on a square lattice and its application to the cuprous oxides, Rev. Mod. Phys. 63, 1 (1991).

[8] P. Fazekas, Lecture Notes on Electron Correlation and Magnetism, Series in Modern Condensed Matter Physics (World Scientific, Singapore, 1999).

[9] A. Reischl, E. Müller-Hartmann, and G. S. Uhrig, Systematic mapping of the Hubbard model to the generalized $t-J$ model, Phys. Rev. B 70, 245124 (2004).

[10] S. A. Hamerla, S. Duffe, and G. S. Uhrig, Derivation of the $t-J$ model for finite doping, Phys. Rev. B 82, 235117 (2010).

[11] M. Hafez-Torbati, N. A. Drescher, and G. S. Uhrig, From gapped excitons to gapless triplons in one dimension, Eur. Phys. J. B 88, 3 (2015).

[12] V. Baltz, A. Manchon, M. Tsoi, T. Moriyama, T. Ono, and Y. Tserkovnyak, Antiferromagnetic spintronics, Rev. Mod. Phys. 90, 015005 (2018).

[13] G. A. Sawatzky and J. W. Allen, Magnitude and Origin of the Band Gap in NiO, Phys. Rev. Lett. 53, 2339 (1984).

[14] P. Němec, M. Fiebig, T. Kampfrath, and A. V. Kimel, Antiferromagnetic opto-spintronics, Nat. Phys. 14, 229 (2018).

[15] A. Kirilyuk, A. V. Kimel, and T. Rasing, Ultrafast optical manipulation of magnetic order, Rev. Mod. Phys. 82, 2731 (2010).

[16] J. Zaanen, G. A. Sawatzky, and J. W. Allen, Band Gaps and Electronic Structure of Transition-Metal Compounds, Phys. Rev. Lett. 55, 418 (1985).
[17] A. Qaiumzadeh, I. A. Ado, R. A. Duine, M. Titov, and A. Brataas, Theory of the Interfacial Dzyaloshinskii-Moriya Interaction in Rashba Antiferromagnets, Phys. Rev. Lett. 120, 197202 (2018).

[18] G. Sangiovanni, A. Toschi, E. Koch, K. Held, M. Capone, C. Castellani, O. Gunnarsson, S.-K. Mo, J. W. Allen, H.-D. Kim, A. Sekiyama, A. Yamasaki, S. Suga, and P. Metcalf, Static versus dynamical mean-field theory of Mott antiferromagnets, Phys. Rev. B 73, 205121 (2006).

[19] X. Wang, E. Gull, L. de' Medici, M. Capone, and A. J. Millis, Antiferromagnetism and the gap of a Mott insulator: Results from analytic continuation of the self-energy, Phys. Rev. B 80, 045101 (2009).

[20] L. Fratino, P. Sémon, M. Charlebois, G. Sordi, and A.-M. S. Tremblay, Signatures of the Mott transition in the antiferromagnetic state of the two-dimensional Hubbard model, Phys. Rev. B 95, 235109 (2017).

[21] T. Satoh, S.-J. Cho, R. Iida, T. Shimura, K. Kuroda, H. Ueda, Y. Ueda, B. A. Ivanov, F. Nori, and M. Fiebig, Spin Oscillations in Antiferromagnetic NiO Triggered by Circularly Polarized Light, Phys. Rev. Lett. 105, 077402 (2010).

[22] T. Kampfrath, A. Sell, G. Klatt, A. Pashkin, S. Mährlein, T. Dekorsy, M. Wolf, M. Fiebig, A. Leitenstorfer, and R. Huber, Coherent terahertz control of antiferromagnetic spin waves, Nat. Photonics 5, 31 (2011).

[23] D. Bossini, A. M. Kalashnikova, R. V. Pisarev, T. Rasing, and A. V. Kimel, Controlling coherent and incoherent spin dynamics by steering the photoinduced energy flow, Phys. Rev. B 89, 060405(R) (2014).

[24] D. Bossini, S. Dal Conte, Y. Hashimoto, A. Secchi, R. V. Pisarev, Th. Rasing, G. Cerullo, and A. V. Kimel, Macrospin dynamics in antiferromagnets triggered by sub-20 femtosecond injection of nanomagnons, Nat. Commun. 7, 10645 (2016).

[25] Y. Hashimoto, D. Bossini, T. H. Johansen, E. Saitoh, A. Kirilyuk, and T. Rasing, Frequency and wavenumber selective excitation of spin waves through coherent energy transfer from elastic waves, Phys. Rev. B 97, 140404(R) (2018).

[26] D. Bossini, S. Dal Conte, G. Cerullo, O. Gomonay, R. V. Pisarev, M. Borovsak, D. Mihailovic, J. Sinova, J. H. Mentink, T. Rasing, and A. V. Kimel, Laser-driven quantum magnonics and terahertz dynamics of the order parameter in antiferromagnets, Phys. Rev. B 100, 024428 (2019).

[27] H.-h. Chou and H. Y. Fan, Effect of antiferromagnetic transition on the optical-absorption edge in $\mathrm{MnO}, \alpha-\mathrm{MnS}$, and $\mathrm{CoO}$, Phys. Rev. B 10, 901 (1974). 
[28] J. Diouri, J. P. Lascaray, and M. El Amrani, Effect of the magnetic order on the optical-absorption edge in $\mathrm{Cd}_{1-\mathrm{x}} \mathrm{Mn}_{\mathrm{x}} \mathrm{Te}$, Phys. Rev. B 31, 7995 (1985).

[29] K. Ando, K. Takahashi, T. Okuda, and M. Umehara, Magnetic circular dichroism of zinc-blende-phase MnTe, Phys. Rev. B 46, 12289 (1992).

[30] D. Bossini, M. Terschanski, F. Mertens, G. Springholz, A. Bonanni, G. S. Uhrig, and M. Cinchetti, Exchange-mediated magnetic blue-shift of the band-gap energy in the antiferromagnetic semiconductor MnTe, New J. Phys. 22, 083029 (2020).

[31] C. Ferrer-Roca, A. Segura, C. Reig, and V. Muñoz, Temperature and pressure dependence of the optical absorption in hexagonal MnTe, Phys. Rev. B 61, 13679 (2000).

[32] W. Szuszkiewicz, E. Dynowska, B. Witkowska, and B. Hennion, Spin-wave measurements on hexagonal MnTe of NiAs-type structure by inelastic neutron scattering, Phys. Rev. B 73, 104403 (2006).

[33] S. Mu, R. P. Hermann, S. Gorsse, H. Zhao, M. E. Manley, R. S. Fishman, and L. Lindsay, Phonons, magnons, and lattice thermal transport in antiferromagnetic semiconductor MnTe, Phys. Rev. Materials 3, 025403 (2019).

[34] J. Hubbard, Electron correlations in narrow energy bands, Proc. R. Soc., London, Ser. A 276, 238 (1963).

[35] G. Rohringer, H. Hafermann, A. Toschi, A. A. Katanin, A. E. Antipov, M. I. Katsnelson, A. I. Lichtenstein, A. N. Rubtsov, and K. Held, Diagrammatic routes to nonlocal correlations beyond dynamical mean field theory, Rev. Mod. Phys. 90, 025003 (2018).

[36] P. R. C. Kent, M. Jarrell, T. A. Maier, and T. Pruschke, Efficient calculation of the antiferromagnetic phase diagram of the three-dimensional Hubbard model, Phys. Rev. B 72, 060411(R) (2005).

[37] R. Staudt, M. Dzierzawa, and A. Muramatsu, Phase diagram of the three-dimensional Hubbard model at half filling, Eur. Phys. J. B 17, 411 (2000).

[38] M. Ulmke, V. Janiš, and D. Vollhardt, Anderson-Hubbard model in infinite dimensions, Phys. Rev. B 51, 10411 (1995).

[39] M. Jarrell, Hubbard Model in Infinite Dimensions: A Quantum Monte Carlo Study, Phys. Rev. Lett. 69, 168 (1992).

[40] T. Pruschke and R. Zitzler, From Slater to Mott-Heisenberg physics: The antiferromagnetic phase of the Hubbard model, J. Phys.: Condens. Matter 15, 7867 (2003).

[41] M. Caffarel and W. Krauth, Exact Diagonalization Approach to Correlated Fermions in Infinite Dimensions: Mott Transition and Superconductivity, Phys. Rev. Lett. 72, 1545 (1994).

[42] S. Doniach, The Kondo lattice and weak antiferromagnetism, Physica B+C (Amsterdam) 91, 231 (1977).

[43] P. Fulde, V. Zevin, and G. Zwicknagl, Model for heavy-fermion behavior of $\mathrm{Nd}_{1.8} \mathrm{Ce}_{0.2} \mathrm{CuO}_{4}$, Z. Phys. B: Condens. Matter 92, 133 (1993).

[44] K. Held and D. Vollhardt, Electronic Correlations in Manganites, Phys. Rev. Lett. 84, 5168 (2000).

[45] C. Zener, Interaction between the $d$-shells in the transition metals. II. ferromagnetic compounds of manganese with perovskite structure, Phys. Rev. 82, 403 (1951).

[46] P. W. Anderson and H. Hasegawa, Considerations on double exchange, Phys. Rev. 100, 675 (1955).

[47] P. G. de Gennes, Effects of double exchange in magnetic crystals, Phys. Rev. 118, 141 (1960).
[48] E. Pavarini, E. Koch, F. Anders, and M. Jarrell, Correlated Electrons: From Models to Materials (Forschungszentrum Jülich, Jülich, Germany, 2012).

[49] E. Müller-Hartmann and E. Dagotto, Electronic Hamiltonian for transition-metal oxide compounds, Phys. Rev. B 54, R6819 (1996).

[50] One notes that we have denoted the local spin by $S$ while in Ref. [49] it is denoted by $S-1 / 2$.

[51] Y. Varshni, Temperature dependence of the energy gap in semiconductors, Physica (Amsterdam) 34, 149 (1967).

[52] X. Zhu, Q. Lian, P. Zhang, W. Bai, K. Tang, L. Zhu, J. Yang, Y. Zhang, X. Tang, and J. Chu, Photoluminescence spectra of the $\mathrm{Mn}^{2+} d-d$ multiplets transitions of zinc-blende MnTe epitaxial films: laser and deuterium lamp excitation study, Opt. Lett. $\mathbf{4 3}$ 5547 (2018).

[53] W. Szuszkiewicz, B. Hennion, B. Witkowska, E. Łusakowska, and A. Mycielski, Neutron scattering study of structural and magnetic properties of hexagonal MnTe, Phys. Status Solidi C 2, 1141 (2005).

[54] S. J. Youn, B. I. Min, and A. J. Freeman, Crossroads electronic structure of MnS, MnSe, and MnTe, Phys. Status Solidi B 241, 1411 (2004).

[55] G. Yin, J.-X. Yu, Y. Liu, R. K. Lake, J. Zang, and K. L. Wang, Planar Hall Effect in Antiferromagnetic MnTe Thin Films, Phys. Rev. Lett. 122, 106602 (2019)

[56] E. Müller-Hartmann, Correlated fermions on a lattice in high dimensions, Z. Phys. B: Condens. Matter 74, 507 (1989).

[57] K. Haule and G. Kotliar, Optical conductivity and kinetic energy of the superconducting state: A cluster dynamical mean field study, EPL (Europhys. Lett.) 77, 27007 (2007).

[58] J. Vučičević, T. Ayral, and O. Parcollet, TRILEX and $G W+$ EDMFT approach to $d$-wave superconductivity in the Hubbard model, Phys. Rev. B 96, 104504 (2017).

[59] K. Walther, Ultrasonic relaxation at the Néel temperature and nuclear acoustic resonance in MnTe, Solid State Commun. 5, 399 (1967).

[60] D. Kriegner, H. Reichlova, J. Grenzer, W. Schmidt, E. Ressouche, J. Godinho, T. Wagner, S. Y. Martin, A. B. Shick, V. V. Volobuev, G. Springholz, V. Holý, J. Wunderlich, T. Jungwirth, and K. Výborný, Magnetic anisotropy in antiferromagnetic hexagonal MnTe, Phys. Rev. B 96, 214418 (2017).

[61] D. Bossini, K. Konishi, S. Toyoda, T. Arima, J. Yumoto, and M. Kuwata-Gonokami, Femtosecond activation of magnetoelectricity, Nat. Phys. 14, 370 (2018).

[62] K. Gillmeister, D. Golež, C. T. Chiang, N. Bittner, Y. Pavlyukh, J. Berakdar, P. Werner, and W. Widdra, Ultrafast coupled charge and spin dynamics in strongly correlated $\mathrm{NiO}$, Nat. Commun. 11, 4095 (2020).

[63] M. Potthoff and W. Nolting, Surface metal-insulator transition in the Hubbard model, Phys. Rev. B 59, 2549 (1999).

[64] Y. Song, R. Wortis, and W. A. Atkinson, Dynamical mean field study of the two-dimensional disordered Hubbard model, Phys. Rev. B 77, 054202 (2008).

[65] M. Snoek, I. Titvinidze, C. Tóke, K. Byczuk, and W. Hofstetter, Antiferromagnetic order of strongly interacting fermions in a trap: real-space dynamical mean-field analysis, New J. Phys. 10, 093008 (2008).

[66] M. Hafez-Torbati and W. Hofstetter, Artificial SU(3) spin-orbit coupling and exotic Mott insulators, Phys. Rev. B 98, 245131 (2018). 
[67] M. Hafez-Torbati and W. Hofstetter, Competing charge and magnetic order in fermionic multicomponent systems, Phys. Rev. B 100, 035133 (2019).

[68] M. Hafez-Torbati, J.-H. Zheng, B. Irsigler, and W. Hofstetter, Interaction-driven topological phase transitions in fermionic SU(3) systems, Phys. Rev. B 101, 245159 (2020).

[69] M. Ebrahimkhas, M. Hafez-Torbati, and W. Hofstetter, Lattice symmetry and emergence of antiferromagnetic quantum Hall states, Phys. Rev. B 103, 155108 (2021).
[70] N. Furukawa, Transport properties of the Kondo lattice model in the limit $S=\infty$ and $D=\infty$, J. Phys. Soc. Jpn. 63, 3214 (1994).

[71] M. J. Calderón and L. Brey, Monte Carlo simulations for the magnetic phase diagram of the double-exchange Hamiltonian, Phys. Rev. B 58, 3286 (1998).

[72] R. Peters and T. Pruschke, Relevance of quantum fluctuations in the Anderson-Kondo model, New J. Phys. 8, 127 (2006).

[73] A. Fetter and J. Walecka, Quantum Theory of Many-Particle Systems, Dover Books on Physics (Dover, Mineola, NY, 2012). 